\title{
A Ground Truth Correspondence Measure for Benchmarking
}

\author{
Johan Karlsson and Anders Ericsson \\ Centre for Mathematical Sciences \\ Lund University \\ johank@maths.lth.se, anderse@maths.lth.se
}

\begin{abstract}
Automatic localisation of correspondences for the construction of Statistical Shape Models from examples has been the focus of intense research during the last decade. Several algorithms are available and benchmarking is needed to rank the different algorithms. Prior work has focused on evaluating the quality of the models produced by the algorithms by measuring compactness, generality and specificity. In this paper problems with these standard measures are discussed. We propose that a ground truth correspondence measure $(\mathrm{gcm})$ is used for benchmarking and in this paper benchmarking is performed on several state of the art algorithms. Minimum Description Length (MDL) with a curvature cost comes out as the winner of the automatic methods. Hand marked models turn out to be best but a semi-automatic method is shown to lie in between the best automatic method and the hand built models in performance.
\end{abstract}

\section{Introduction}

In recent years there has been a lot of work on automatic construction of Shape Models. There are several different algorithms for this automatic model construction. The algorithms usually locate parameterisations of the shapes in the training set to get correspondences between the shapes.

Attempts have been made to locate correspondences on shapes using shape features, such as high curvature [13]. Many have stated the correspondence problem as an optimisation problem $[1,2,7,9,8,11,12,15,17]$. Minimum Description Length, (MDL) [6], is a paradigm that has been used in many different applications, often in connection with model optimisation. In recent papers $[6,9,14]$ this paradigm is used to locate a dense correspondence between shapes. There have been some recent interesting papers including code on the problem of matching one point set to another, $[3,4,19]$. However these algorithms only match one shape to another, instead of working with the training set as a whole.
In short the field has matured and there are many algorithms available. So there is a need for benchmarking of these algorithms. In recent years a similar development has taken place in the field of stereo [16].

In order to evaluate these algorithms, different measures of the quality of the parameterisations and the resulting models have been used. If the model is to be used for a specific purpose, such as segmentation of the heart in scintigrams, the choice of algorithm should be made using a criterion based on the application. For a more general evaluation of shape model quality the standard measures are compactness, specificity and generality [5]. It is also common to evaluate correspondences subjectively by plotting the shapes with some corresponding points marked.

In this paper problems with these standard general model quality measures, namely compactness, generality and specificity, are discussed. We show that especially specificity and compactness do not succeed in measuring what they attempt to measure. With practical experiments we also show that the standard measures do not correlate well with correspondence quality. Also these measures are not quantitative in the sense that they do not assign a single number to describe the quality of the shape model.

What should be considered as a shape model of high quality is highly dependent on the application. For example the model that performs best on segmentation might not be the model that performs best on classification.

The qualities that the standard measures attempt to measure are often, but certainly not always, important. However, even when they are important, as we will see, it is problematic to actually measure them.

For most applications high quality of the correspondences is desirable. A shape model built from correct correspondences is a model that correctly describes the shape variation within the class, whereas, as will be shown, a simplified model can get excellent measure of specificity, generality and compactness, but relevant shape information may have been lost.

We propose that a ground truth correspondence measure $(\mathrm{gcm})$, measuring the quality of the correspondences at 
important locations, is used for measuring correspondence quality and that this is used for benchmarking.

Verification using ground truth is an established method in computer vision, see for example the CAVIAR project [10].

The four major contributions in this paper are: (i) It is shown that former shape model measures have severe weaknesses. (ii) A correspondence quality measure $(\mathrm{gcm})$ is proposed and it is shown that $\mathrm{gcm}$ together with a database of ground truth correspondences is well suited for benchmarking algorithms for correspondence localisation. (iii) A database of datasets and matlab code for evaluating algorithms is published. (iv) Benchmarking of several state of the art algorithms is presented and MDL with curvature cost comes out as the winner of the automatic methods. (v) Contrary to former results handmade models are shown to be the best and a semiautomatic algorithm is proposed that outperfroms all of the automatic algorithms.

\section{Shape Model Preliminaries}

When analysing a set of $n_{s}$ similar shapes, it is convenient and usually effective to describe them using Statistical Shape Models. The shapes are typically boundaries of some objects and are commonly defined by landmarks, curves or surfaces. In this paper we treat the shapes as $n$-dimensional manifolds. Specifically we consider one-dimesional manifolds, i.e. curves, but the measures discussed work equivalently for $n$-dimesional manifolds, for example surfaces. After the shapes $\mathbf{x}_{i}\left(i=1 \ldots n_{s}\right)$ have been aligned and normalised to the same size, a principal component analysis of the covariance matrix for the shapes is performed. A shape from the shape class can now be described by a linear shape model of the form,

$$
\mathbf{x}=\overline{\mathbf{x}}+\mathbf{\Phi} \mathbf{b}+\mathbf{e},
$$

where $\overline{\mathbf{x}}$ is the mean shape, the columns of $\boldsymbol{\Phi}$ describe a set of orthogonal modes of shape variation and $\mathbf{b}$ is the vector of shape parameters for the shape and $\mathbf{e}$ is the residual.

\section{Measuring Model Quality}

Generality By generality is meant how well the model can generalise to formerly unseen shapes. The model should be able to describe all shapes of the class and not only those of the training set. This is measured by doing leave one out tests, where a model is built by using all but one of the shapes in the training set. This model tries to describe the left out shape. The error in [5] for one left out shape is the norm of the difference between the modeled shape and the true shape. Generality is measured as the mean over all $n_{s}$ left out shapes. Usually this is plotted over the number of modes used by the approximating

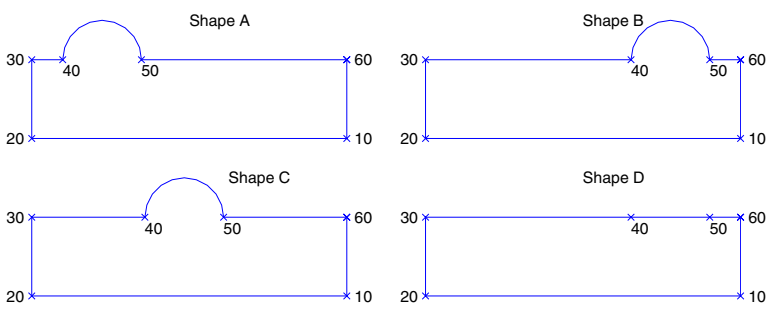

Figure 1. Two models are built from shape $A$ and shape $B$. It turns out that the model that generates shapes like shape $D$ has a better specificity than the model that generates shapes like shape $C$, but shape $D$ does not belong to the shape class whereas shape $C$ does.

model. The expression for generality is

$$
G\left(n_{m}\right)=\frac{1}{n_{s}} \sum_{j=1}^{n_{s}}\left\|\mathbf{x}_{j}-\mathbf{x}_{j}^{\prime}\left(n_{m}\right)\right\|^{2}
$$

where $\mathbf{x}_{j}$ is the left out shape and $\mathbf{x}_{j}^{\prime}\left(n_{m}\right)$ is the attempted description using the model with $n_{m}$ modes. One problem with measuring generality is that the parameterisation of the left out shape is unknown. This is often solved by letting the shape be included in the correspondence localisation, but this leads to an underestimation of the error.

Specificity It is in general desirable that a shape model is specific. A specific model can only represent shapes from the shape class for valid parameter values. This has been measured by generating a large amount $(\mathrm{N})$ of shapes by picking random parameter values to the model. Each sample shape is then compared to the most similar shape in the training set. A quantitative measure for this [5] is:

$$
S\left(n_{m}\right)=\frac{1}{N} \sum_{j=1}^{N}\left\|\mathbf{y}_{j}-\mathbf{y}_{j}^{\prime}\left(n_{m}\right)\right\|^{2}
$$

where $\mathbf{y}_{j}^{\prime}$ are shape examples generated by the model and $\mathbf{y}_{j}$ is the nearest member of the training set to $\mathbf{y}_{j}^{\prime}$ and $n_{m}$ is the number of modes used to create the samples.

In Figure 1 a number of boxbump shapes are shown. For shape A, B and C landmark 40 is at the left corner of the bump, landmark 50 is at the right corner of the bump and the landmarks 10, 20, 30 and 60 are at the corners of the rectangle in clockwise order. Between these landmarks the landmarks are placed by arc length parameterisation. Shape $\mathrm{D}$ is constructed by taking shape $\mathrm{B}$ and projecting down the bump onto the rectangle. Assume that two different models of boxbumps is built from shape A and shape B. Shape C 
also belongs to the class of box bumps but shape D does not. Say that model 1 generates shapes like Shape $\mathrm{C}$ and model 2 generates shapes like shape D. Look at the terms in (2). For model 2 the term that comes from shape $\mathrm{D}$ will be less than the term for model 1 that comes from shape $\mathrm{C}$. This will imply that model 2 is more specific than model 1 , but shape $\mathrm{C}$ belongs to the shape class whereas shape $\mathrm{D}$ does not.

The standard specificity measure can give the same error for shapes that belong to the shape class (but are between two shapes of the training set) as it does for shapes that do not belong to the shape class.

Compactness A compact model is a model that represents all shapes of the class with as little variance as possible in the shape variation modes and preferably with few modes. A measure of compactness is the sum of variances of the model [5],

$$
C\left(n_{m}\right)=\sum_{j=1}^{n_{m}} \lambda_{j}
$$

where $\lambda_{j}$ is the variance in shape mode $j$ and $C\left(n_{m}\right)$ is the compactness using $n_{m}$ number of modes. If the curve for one model is below or equal to the curve for another model for all $n_{m}$ and lower for some $n_{m}$, the model represented by the lower curve is said to be more compact [5]. One problem with this measure is that it does not take into consideration the value of gathering the varation in few modes. Also a common situation when measuring generality, specificity and compacness is that the curves for different models intersect and in this case it is not possible to choose which model is of higher quality.

\section{Ground Truth Correspondence Measure}

In order to measure the quality of the correspondences produced by an algorithm for automatic correspondence localisation, datasets with manually located landmarks and synthetic datasets with known corresponding points can be used. For synthetic examples these marks are exact but for manually placed marks there is of course a subjective element and also the introduction of a small error is inevitable.

Let the parameterisations $\gamma_{i}$ of the shapes $\mathbf{x}_{i}$ be optimised by the algorithm that is to be evaluated. Then, for every shape $\mathbf{x}_{i}\left(i=1, \ldots, n_{s}\right)$ together with its ground truth points $\mathbf{g}_{i j}\left(j=1, \ldots, n_{g}\right)$, find $t_{i j}$ so that $\mathbf{x}_{i}\left(\gamma_{i}\left(t_{i j}\right)\right)=$ $\mathbf{g}_{i j}$. This means that the parameter values that correspond to the ground truth points on the shape are calculated. Formally $t_{i j}=\gamma_{i}^{-1}\left(\mathbf{x}_{i}^{-1}\left(\mathbf{g}_{i j}\right)\right)$. Now, for every shape $\mathbf{x}_{k}$ ( $1 \leq k \leq n_{s}, k \neq i$ ) use the same parameter values $t_{i j}$. The points produced should be close to the ground truth points of this shape, if the parameterisation functions represent correspondences of high quality. That is, $\mathbf{x}_{k}\left(\gamma_{k}\left(t_{i j}\right)\right)$ should be close to $\mathbf{g}_{k j}$. This is measured as the mean distance in the metric $d$ over all shapes in the dataset.

$$
\begin{gathered}
g c m=\frac{1}{n_{s}\left(n_{s}-1\right) n_{g}} \sum_{i=1}^{n_{s}} \sum_{j=1}^{n_{g}} \sum_{k \in K} e_{i j k} \\
e_{i j k}=d\left(\mathbf{x}_{k}\left(\gamma_{k}\left(\gamma_{i}^{-1}\left(\mathbf{x}_{i}^{-1}\left(\mathbf{g}_{i j}\right)\right)\right)\right), \mathbf{g}_{k j}\right), \\
K=\left\{1, \ldots, i-1, i+1, \ldots, n_{s}\right\} .
\end{gathered}
$$

Finally we get,

$g c m=\frac{1}{n_{s}\left(n_{s}-1\right) n_{g}} \sum_{i=1}^{n_{s}} \sum_{j=1}^{n_{g}} \sum_{k \in K} d\left(\mathbf{x}_{k}\left(\gamma_{k}\left(t_{i j}\right)\right), \mathbf{g}_{k j}\right)$,

where $t_{i j}$ is the parameterisation parameter value for the ground truth point $j$ on shape $i$. The constant $n_{s}$ is the number of shapes and $n_{g}$ is the number of ground truth points. Any metric could be used, but here $d(a, b)$ means the length of the shortest path between the point $a$ and the point $b$ along the shape, which has been normalised to have area one. Locally (usually also globally) the shortest path is a geodesic. Apart from the advantage of measuring the error along the shape, this also gives scale invariance. On curves the metric $d$ corresponds to the arclength distance on curves normalised to length one.

Note that in case of intersections of the shape the path is not allowed to use the intersection as a short cut.

Ground Truth Correspondence Measure with Mahalanobis Distance Due to the subjective nature of choosing ground truth points on natural shapes, statistics about the ground truth points could be taken into account. Let a number of people mark ground truth points on the same dataset. Means and variances can then be calculated and the norm used to calculate $\mathrm{gcm}$ can then be the Mahalanobis normalised geodesic distance,

$$
\begin{gathered}
g c m=\frac{1}{n_{s}\left(n_{s}-1\right) n_{g}} \sum_{i=1}^{n_{s}} \sum_{j=1}^{n_{g}} \sum_{k \in K} \frac{d\left(\mathbf{x}_{k}\left(\gamma_{k}\left(t_{i j}\right)\right), \overline{\mathbf{g}}_{k j}\right)}{\sigma_{k j}} \\
K=\left\{1, \ldots, i-1, i+1, \ldots, n_{s}\right\}
\end{gathered}
$$

where $\overline{\mathbf{g}}_{k j}$ is the mean and $\sigma_{k j}$ is the standard deviation for landmark $j$ on shape $k$.

\section{Experimental Validation of $\mathrm{gcm}$}

The first experiment starts from correct correspondences and then optimises the parameterisations so as to minimise the description length. Synthetic box bump shapes have been used for this test, since the true correspondences are known here. The value of the description length (DL) and the ground truth correspondence measure $(\mathrm{gcm})$ over the number of iterations is plotted in Figure 2. 

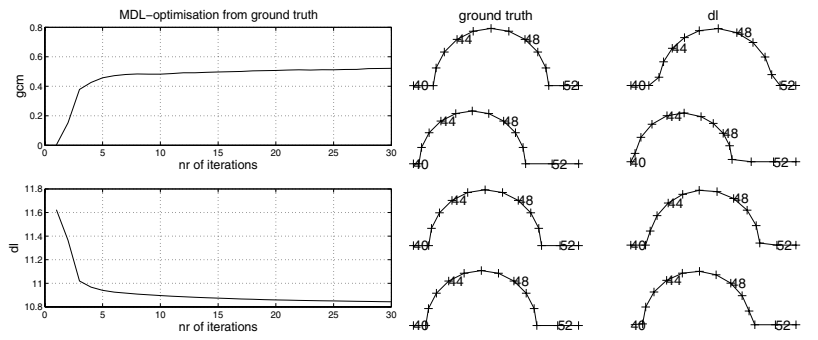

Figure 2. To the left Gcm and dl plotted over number of iterations. To the right true correspondences and optimised correspondences.

It is interesting to note here that the $\mathrm{gcm}$ increases (gets worse) as the description length decreases (gets better). The minimum, when the parameterisation is optimised with description length as goal function, does not correspond to true correspondences. In Figure 2 it can be seen that minimising the description length from true correspondences has resulted in worse correspondences. The plot shows 4 boxbump shapes zoomed in on the bump.
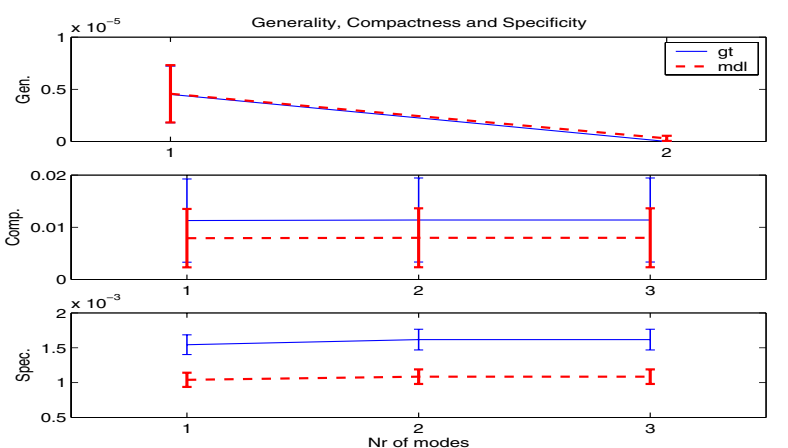

Figure 3. Generalisation, compactness and specificity of the ground truth boxbump model and the optimised box bump model.

In Figure 3 the compactness and specificity measures indicate that the optimised model has higher quality. In this case we started from ground truth and as can be seen in Figure 2 the correspondences are worse for the optimised model. So we can conclude that compactness and specificity do not correlate with correct correspondences. As an extreme example it is possible to get perfect compactness, specificity and generality (zero) by placing all landmarks in one point on all shapes.

The problem with the specificity measure is that if the training set is limited (which is often the case) it can not be assumed that all shapes of a class are close to one of the shapes in the training set.

Summing up this experiment, the conclusion is that although minimising the description length is a good method, it does not measure what we really wish to optimise and in this case it fails to recognise the true optimum. We also conclude that compactness and specificity can not, in general, decide which model is the best.

Summing up this experiment, the conclusion is that although minimising the description length is a good method for finding approximate correspondences, in this experiment it fails to locate the correct correspondences. We also conclude that compactness and specificity do not correlate with the quality of the correspondences in general.

In the second experiment silhouette shapes initialised with arclength parameterisation were used. The parameterisations were optimised with respect to MDL [6] until convergence (40 iterations). Then we continue the optimisation with respect to MDL plus a curvature cost [18] until convergence (another 40 iterations).

Figure 4 shows the resulting correspondences on the part of the shapes corresponding to the eye. The plots show landmark 25 to 40 . Anatomically this shows the end of the forehead and the beginning of the nose of a person looking to the left. The nose begins approximately at landmark 34 in the bottom row. The correspondences are clearly better when using curvature. Other parts of the curves are similar. The Figure 6 shows how gcm decreases when curvature is

\section{dl}

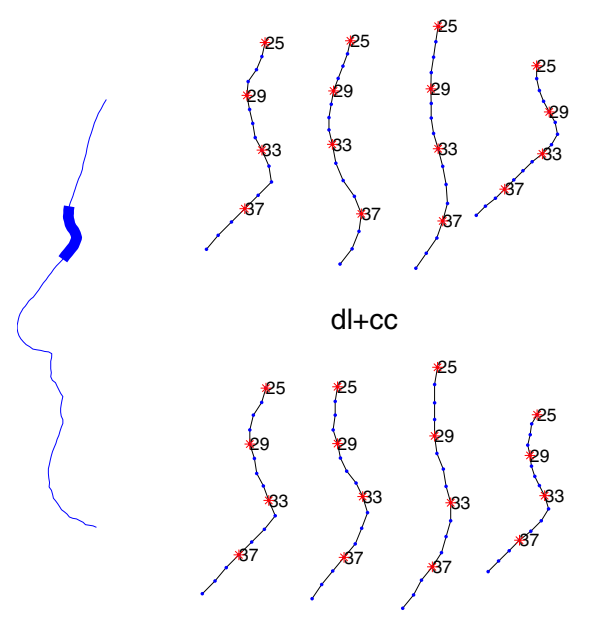

Figure 4. Corresponding landmarks on parts of silhouettes. To the left the part zoomed in on is marked bold on a whole silhouette.

added. The middle plot shows how DL first decreases as it is minimised, but then when DL + curvature cost is minimised in the second part DL increases. So gcm captures an improvement in correspondences that DL misses. In Figure 5 it can be seen how the measures of generality, compactness and specificity all indicate that the model without curvature cost has higher quality. So the standard measures can not be used to measure correspondence quality. This experiment shows that $\mathrm{gcm}$ captures an improvement in cor- 


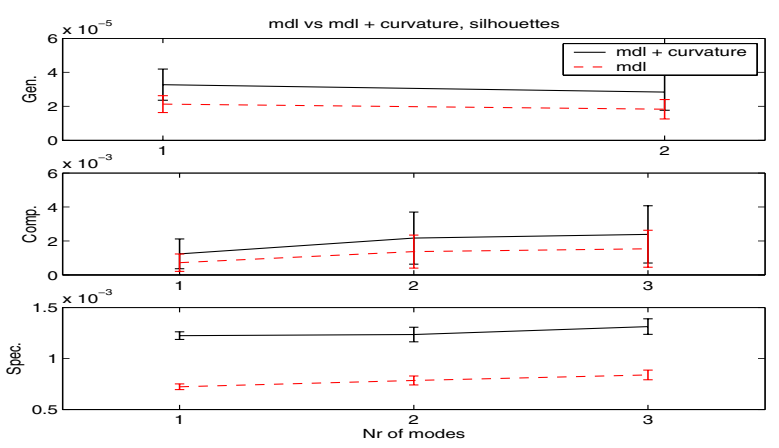

Figure 5. Generalisation, compactness and specificity of the DL silhouette model and the DL + curvature cost silhouette model.
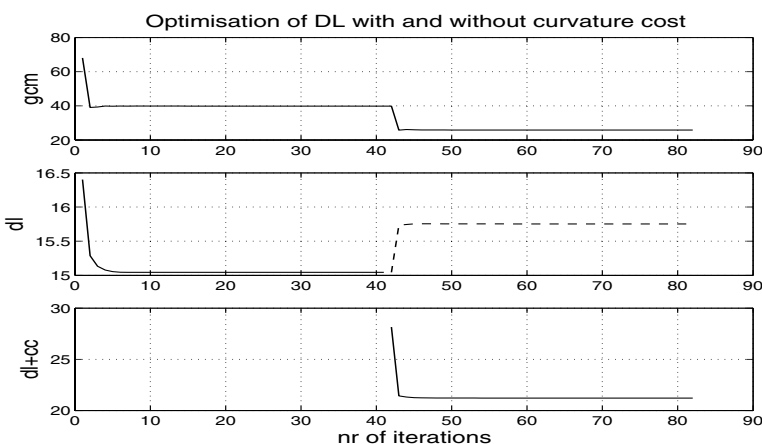

Figure 6. Optimisation of $\mathrm{DL}$ and $\mathrm{DL}+$ curvature cost for 4 silhouettes.

respondences that generality, compactness, specificity and DL all miss. This indicates that they can not be used for evaluating correspondence quality.

\section{Benchmarking using gcm}

First a database of eight shape classes was built. The first five shape classes (sharks, birds, flightbirds, rats, forks) are curves from the database used in [17] and each of these shape classes consists of 13-23 shapes. The sixth and eighth shape classes are the silhouette- and boxbump-shapes from the previous section. The seventh shape class is a hand segmented from a video sequence. The boxbumps are synthetic with 61 ground truth points on each shape. The ground truth correspondences on the first seven data sets 10-21 landmarks were manually marked. This database together with code can be downloaded from www. maths. lth.se/matematiklth/vision/ddd

The following algorithms have been benchmarked using gcm: Arclength, MDL [6], MDL with curvature (MDL+Cur) [18], MDL with parameterisation invariant scalar product (MDL+Par) [14] , aias [8] , aias with MDL, reparameterisation by minimising Euclidean distance (eucl), reparameterisation by minimising Euclidean and
Curvature distance (eucl+cur). Also handmade models of all the datasets were built by a different person than the one marking ground truth. This was done without knowing which anatomical points were used as ground truth.

All tests were performed with 128 landmarks, 40 iterations and 7 reparameterisation control nodes for all the automatic algorithms. Table 1 shows the remaining percentage of $\mathrm{gcm}$ after optimising from arclength (100\% means equal $\mathrm{gcm}$ as arclength parameterisation and $0 \%$ means perfect correspondences according to $\mathrm{gcm}$ ).

MDL with a curvature cost added comes out as the winning algorithm. There is no algorithm that is best on all datasets and no algorithm gives as good correspondences as the correspondences marked manually.

Ground truth points have been marked for the shark dataset by 11 people. The geodesic Mahalanobis gcm was calculated for the algorithms in the same order as Table 1 and the percentage left were: 25.4, 22.2, 27.7, 25.3, 36.4, 48.4, 24.5 and 19.8. Again MDL+Cur comes out as the winner of the automatic methods but the handmade is best.

For the winning algorithm $\mathrm{gcm}$ was then used to pick optimal parameter values, such as number of landmarks and number of parameterisation nodes, using the shark dataset. This resulted in even better results also on the other datasets.

Semi-Automatic Algorithm Since handmade models are best, a semi-automatic algorithm was tested. Five shapes were manually marked and then kept fixed, while the rest of the shapes in the dataset were optimised one by one using DL with curvature cost to fit the five fixed shapes. This resulted in an algorithm better than all the automatic algorithms, see Table 1. Seven control nodes were used for all natural datasets but for the synthetic boxbumps 15 nodes were used. Experiments with 15 nodes for the automatic algorithms results in worse correspondences for all algorithms except eucl and eucl+cur where only slightly better results were obtained.

\section{Summary and Conclusions}

For evaluation of the quality of shape models built from correspondences located automatically there have formerly been a number of standard methods. In this paper problems with these measures are discussed and it is shown that they do not correlate with correspondence quality. We propose a Ground truth Correspondence Measure $(\mathrm{gcm})$ for the evaluation of correspondence quality to be used in benchmarking. It is shown in experiments on different datasets that this measure corresponds well to subjective evaluation of correspondence quality. It is also shown that optimising correspondences using description length initiated with correct correspondences can result in worse correspondences.

In this paper several state of the art algorithms are benchmarked using $\mathrm{gcm}$. In Table 1 it can be seen that MDL+Cur 


\begin{tabular}{|l|c|c|c|c|c|c|c|c|c|c|}
\hline Algorithm & sharks & birds & flightbird & rats & forks & silhouettes & hands & boxbumps & mean & median \\
\hline aias+mdl & $\mathbf{2 5 . 5}^{1}$ & $\mathbf{8 0 . 6}^{1}$ & $62.6^{5}$ & $28.0^{2}$ & $22.7^{4}$ & $42.9^{2}$ & $21.6^{\mathbf{2}}$ & $75.9^{3}$ & $45.0^{3}$ & $35.5^{3}$ \\
MDL+Cur & $26.1^{2}$ & $88.1^{4}$ & $58.3^{2}$ & $29.0^{4}$ & $22.7^{3}$ & $\mathbf{3 2 . 5}^{\mathbf{1}}$ & $\mathbf{1 9 . 4}^{\mathbf{1}}$ & $\mathbf{2 5 . 2}^{\mathbf{1}}$ & $\mathbf{3 7 . 7}^{\mathbf{1}}$ & $\mathbf{2 7 . 5 ^ { \mathbf { 1 } }}$ \\
MDL & $32.9^{5}$ & $90.1^{6}$ & $66.2^{6}$ & $28.6^{3}$ & $22.4^{2}$ & $46.2^{4}$ & $22.3^{3}$ & $30.0^{2}$ & $42.3^{2}$ & $31.4^{2}$ \\
MDL+Par & $29.4^{4}$ & $89.6^{5}$ & $62.4^{4}$ & $\mathbf{2 7 . 3}^{\mathbf{1}}$ & $\mathbf{2 2 . 3 ^ { \mathbf { 1 } }}$ & $42.9^{3}$ & $22.3^{4}$ & $76.6^{4}$ & $46.6^{4}$ & $36.2^{4}$ \\
eucl & $55.9^{6}$ & $85.7^{2}$ & $59.5^{3}$ & $30.8^{5}$ & $25.2^{5}$ & $126.2^{6}$ & $34.7^{6}$ & $118.2^{5}$ & $67.0^{6}$ & $57.7^{6}$ \\
eucl+cur & $27.7^{3}$ & $86.6^{3}$ & $\mathbf{5 6 . 7}^{\mathbf{1}}$ & $33.0^{6}$ & $28.8^{6}$ & $90.0^{5}$ & $22.6^{5}$ & $118.8^{6}$ & $58.0^{5}$ & $44.9^{5}$ \\
\hline semiauto & 22.8 & 76.1 & 50.7 & 24.5 & 21.1 & 26.0 & 17.1 & 16.5 & 31.8 & 23.6 \\
\hline handmade & 18.5 & 73.2 & 27.6 & 13.1 & 11.1 & 21.1 & 14.9 & 0.0 & 22.4 & 16.7 \\
\hline
\end{tabular}

Table 1. Percentage of $\mathrm{gcm}$ error left after optimising from arclength. Upper index indicates rank.

is the best algorithm. There is no algorithm that is best on all datasets and no algorithm gives as good correspondences as the correspondences marked manually. The semiautomatic algorithm is better than the automatic algorithms on all datasets.

In previous work it is often claimed that automatic algorithms give better correspondences than models built by hand. These claims are often supported by measures like generality, specificity and compactness, but we have shown that these measures are not suitable for evaluating correspondences. Measuring $\mathrm{gcm}$, it is concluded that models carefully built by hand are actually very good. In some cases it may not be reasonable to manually mark the full dataset but, as seen, a semi-automatic approach, where only five shapes need to be manually marked, works very well.

\section{Acknowledgments}

We thank H. Thodberg (IMM, DTU) for the silhouettes and B. Kimia et.al. for the images of the sharks, birds, flight birds, rats and forks.

This work has been financed by the SSF sponsored project 'Vision in Cognitive Systems' (VISCOS) and by UMAS and the Swedish Knowledge Foundation through the Industrial $\mathrm{PhD}$ program in Medical Bioinformatics at the Centre for Medical Innovations (CMI) at the Karolinska Institute.

\section{References}

[1] S. Belongie, J. Malik, and J. Puzicha. Shape matching and object recognition using shape contexts. IEEE Trans. Pattern Analysis and Machine Intelligence, 24(24):509-522, 2002.

[2] F. Bookstein. Landmark methods for forms without landmarks: Morphometrics of group differences in outline shape. Medical Image Analysis, 3:225-243, 1999.

[3] H. Chui and A. Rangarajan. A feature registration framework using mixture models. In IEEE Workshop on Mathematical Methods in Biomedical Image Analysis (MMBIA), pages 190-197, 2000.

[4] H. Chui and A. Rangarajan. A new algorithm for non-rigid point matching. In IEEE Conference on Computer Vision and Pattern Recognition (CVPR), volume volume II, pages 44-51, 2000.

[5] R. Davies. Learning Shape: Optimal Models for Analysing Natural Variability. PhD thesis, University of Manchester, 2002.
[6] R. Davies, C. Twining, T. Cootes, J. Waterton, and C. Taylor. A minimum description length approach to statistical shape modeling. IEEE Trans. medical imaging, 21(5):525-537, 2002.

[7] A. Ericsson. Automatic shape modelling and applications in medical imaging. Technical report, Mathematics LTH, Centre for Mathematical Sciences, Box 118, SE-22100, Lund, Sweden, nov 2003.

[8] A. Ericsson and K. Åström. An affine invariant deformable shape representation for general curves. In Proc. 9th Int. Conf. on Computer Vision, Nice, France, pages 1142-1149, Nice, France, 2003.

[9] A. Ericsson and K. Åström. Minimizing the description length using steepest descent. In Proc. British Machine Vision Conference, Norwich, United Kingdom, volume 2, pages 93-102, 2003.

[10] R. Fisher. Caviar project, 2005. Ground truth labelled video sequences.

[11] A. Hill and C. Taylor. Automatic landmark generation for point distribution models. In Proc. British Machine Vision Conference, pages 429-438, 1994.

[12] A. Hill and C. Taylor. A framework for automatic landmark indentification using a new method of nonrigid correspondence. IEEE Trans. Pattern Analysis and Machine Intelligence, 22:241-251, 2000.

[13] C. Kambhamettu and D. Goldgof. Points correspondences recovery in non-rigid motion. In Proc. Conf. Computer Vision and Pattern Recognition, CVPR'92, pages 222-237, 1992.

[14] J. Karlsson, A. Ericsson, and K. Åström. Parameterisation invariant statistical shape models. In Proc. International Conference on Pattern Recognition, Cambridge, UK, 2004.

[15] A. Kotcheff and C. Taylor. Automatic construction of eigenshape models by direct optimization. Medical Image Analysis, 2:303-314, 1998.

[16] D. Scharstein and R. Szeliski. A taxonomy and evaluation of dense two-frame stereo correspondence algorithms. IJCV, 47(1/2/3):7-42, 2002.

[17] T. Sebastian, P. Klein, and B. Kimia. Constructing 2d curve atlases. In IEEE Workshop on Mathematical Methods in Biomedical Image Analysis, pages 70-77, 2000.

[18] H. H. Thodberg and H. Olafsdottir. Adding curvature to minimum description length shape models. In Proc. British Machine Vision Conference, 2003.

[19] Y. Zheng and D. Doermann. Robust point matching for nonrigid shapes: A relaxation labeling based approach. Technical report: Lamp-tr-117, University of Maryland, College Park, 2004. 